\title{
First results of beamtests of a MAPS based ElectroMagnetic calorimeter
}

\author{
G. Nooren* and E. Rocco \\ Address \\ Institute for Subatomic Physics Utrecht University and Nikhef, P.O.B. 80000, 3508 TA Utrecht, \\ the Netherlands
}

E-mail: hoorendnikhef.n]

\begin{abstract}
A prototype of a Si-W EM calorimeter was built with Monolithic Active Pixel Sensors as the active elements. With a pixelsize of $30 \mu \mathrm{m}$ it allows digital calorimetry, i.e. the particles energy is determined by counting pixels, not by measuring the energy deposited. Although of modest size, only 4 Moliere radii wide, it has 39 million pixels and its calibration appears far from trivial. The calorimeter has been tested at DESY (electrons) and at CERN PS and SPS (mixed beams) with energies from 2 to $200 \mathrm{GeV}$. We present the shape of showers caused by electrons and pions, as well as tracks by pions and cosmic muons in unprecedented detail. Preliminary results for energy and position resolution will also be given.
\end{abstract}

11th International Conference on Large Scale Applications and Radiation Hardness of Semiconductor Detectors

3-5 July 2013

Florence, Italy

\footnotetext{
* Speaker.
} 


\section{Introduction}

Fine grained calorimetry was implemented in the past, e.g. in the PAMELA space mission [W] using silicon strip detectors, but is not a common technique. The advantages in tracking and particle identification are obvious. Longitudinally, high granularity allows to determine the shower shape which helps to discriminate showering and non-showering particles. Transversely it is a conditio sine qua non in order to discriminate the photons originating from $\pi^{0}$ decay, from direct photons. This is especially true at forward angles where energies are high and the distance between the photons becomes very small. Such a calorimeter would have a lateral resolution of $1 \mathrm{~mm}$ and a longitudinal segmentation of $1 X_{0}$. This leads to an enormous number of channels, which is only feasible with digital calorimetry. In order for digital calorimetry to work the occupancy per pixel should be less than one. With a particle density of $10^{3} \mathrm{~mm}^{-2}$ in the shower core, one requires very small pixels, in the order of $50-100 \mu \mathrm{m}$.

To fully exploit the geometrical resolution obtainable with this small pixelsize, the Moliere radius of the calorimeter should be as small as possible. This leads to the choice of tungsten as absorber and silicon sensors, whilst minimizing the thickness of the electrical connections. Connecting mm-size pixels to front-end electronics in a classical way (e.g. by bump-bonding) is very costly and has never been shown to work for large surface sensors. The availability of Monolithic Active Pixel Sensors [ []] solves the connection problem between sensor and front-end in an elegant way. Having sensor and front-end electronics on the same chip also reduces the thickness of the construction which leads to smaller effective Moliere radius. A calorimeter based on MAPS was proposed in the context of the CALICE project for ILC [B]. The last decade intense development of MAPS has occured at several places, like RAL (TPAC), IPHC (MIMOSA [田]) and CERN [[]], in view of upgrades of the trackers of STAR [四] and ALICE [ []].

Given the great prospects offered by this technique it was decided to build a prototype and test it with beam [8]. This protype has proven the feasibility of a digital calorimeter [Q]. In particular it has shown that adequate cooling can be achieved with a small Moliere radius. It has provided data from which the resolution and Moliere radius could be determined and which will be used for the study of data volume/flow and data reduction schemes. Calibration and alignment are in progress.

\section{Design}

\subsection{General}

Compared to a conventional sensor, the active pixel sensor dissipates much more heat, typically $0.1 \mathrm{~W} \cdot \mathrm{cm}^{-2}$. It was realised that the power can be transported from the chips to the outside by using the rather good heat conductivity of $\mathrm{W}$, namely $170 \mathrm{~W} / \mathrm{m} / \mathrm{K}(\mathrm{Al}: 237 \mathrm{~W} / \mathrm{m} / \mathrm{K})$. As the tungsten is used as absorber anyway, it can serve the two purposes. To this end the perimeter of the $\mathrm{W}$ plate is connected to cooling elements. The absence of a separate layer of cooling elements leads to a very compact calorimeter, so to a small Moliere radius.

As the chip is not sensitive over its full surface due to the presence of control and output circuitry there is a need for overlap. With the size of MAPS sensors restricted to the typical reticule size of $2 \mathrm{~cm}$ the chance of a shower containing a dead zone would be too high. 


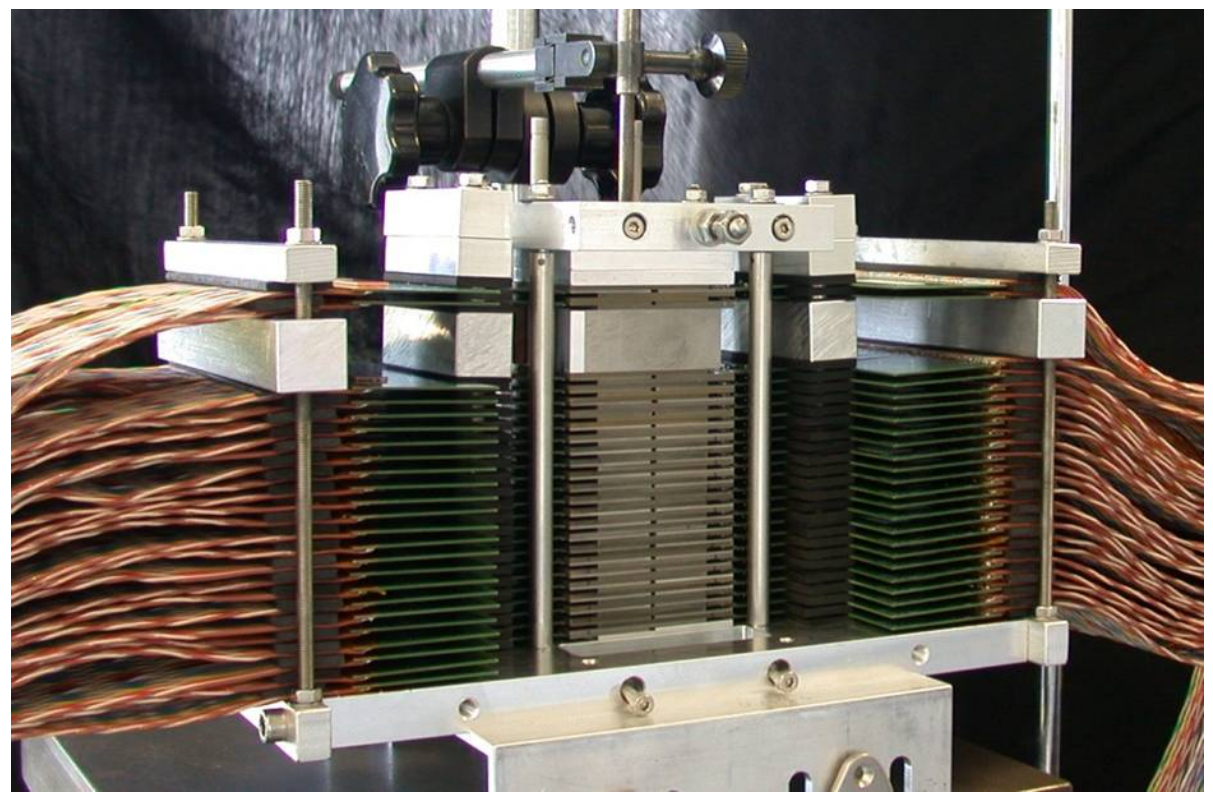

Figure 1: The prototype tower during assembly, before mounting the cooling blocks. The beam direction is from below. Total length in the beam direction is $11 \mathrm{~cm}$, made up of 22 layers, a $20 \mathrm{~mm}$ thick W absorber and 2 additional layers.

The prototype has 24 layers with a thickness of $4 \mathrm{~mm}$ of which the sensor part, including the read-out board, is $1 \mathrm{~mm}$. This leaves an airgap of only $0.1 \mathrm{~mm}$ between the overlapping sensors. The radiation thickness of one layer is $0.97 X_{0}$. The active area of a layer is $4 * 4 \mathrm{~cm}^{2}$, composed of four sensors, while the absorber measures $5 * 5 \mathrm{~cm}^{2}$. The dead zones of the sensors in one layer overlap in one direction only. For ease of construction there remains a deadzone of $0.1 \mathrm{~mm}$ between each pair of chips in the other direction. The Moliere radius calculated from this is $11 \mathrm{~mm}$, which means that the tower is wide enough to study the lateral shower development. The first active layer has ony $0.02 X_{0}$ in front, to act as a charged particle detector. Between layers 21 and $226.7 X_{0}$ of tungsten are placed to obtain a total depth of $28 X_{0}$. At a dissipation of $70 \mathrm{~W}$ in $150 \mathrm{~cm}^{3}$ a heat resistance of $0.3 \mathrm{~K} / \mathrm{W}$ from chip to cooling water was measured.

\subsection{Sensors}

It was decided to build the prototype with available MAPS sensors. The choice is limited: most are too small, like TPAC and many MIMOSA. Apart from TPAC, they were designed with tracker application in mind: small pixels, on-board data reduction. This latter feature is unwanted because it presupposes a certain occupancy, which can be predicted for a tracker, whereas in our case the occupancy is one of the unknowns one wants to measure.

We chose PHASE2/MIMOSA23 from IPHC [ए]]. This is the only full size MAPS ( $640 * 640$ pixels) which allows the continous read-out of all pixels, thanks to four outputs at $160 \mathrm{MHz}$ clock. The $1 \mathrm{MHz}$ rolling shutter implies an integration time of $640 \mu \mathrm{s}$. The resulting low event rate is no problem in the case of test experiments. The small pixel pitch of $30 \mu \mathrm{m}$ allows very fine sampling of the shower core. 

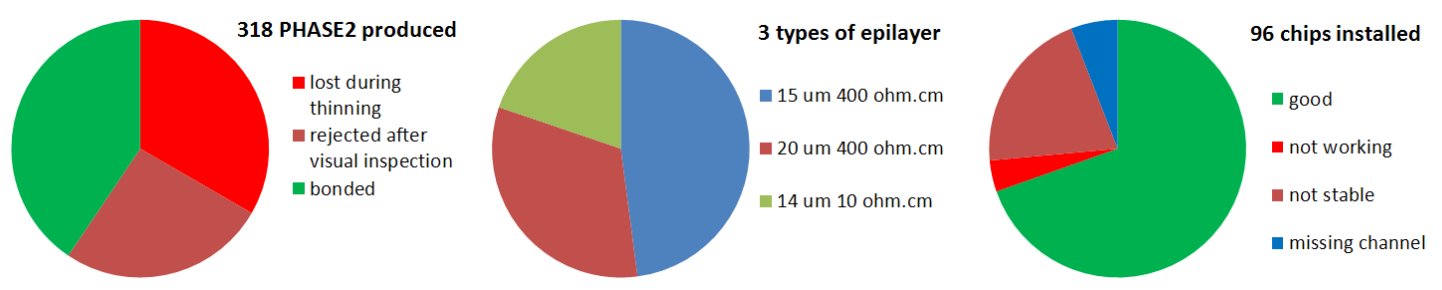

Figure 2: PHASE2 sensorchips from one engineering run: left: fraction of useable sensorchips, centre: distribution of the types of epilayer and right: quality of the installed sensorchips.

An engineering run at $\mathrm{AMS}^{1}$ provided 5 wafers with a high resistivity $(400 \Omega \mathrm{cm})$ epilayer of 15 and $20 \mu \mathrm{m}$ thickness and 1 standard wafer $(10 \Omega \mathrm{cm}, 14 \mu \mathrm{m})$. The former ones were thinned down to $120 \mu \mathrm{m}$, the latter to $180 \mu \mathrm{m}$. The lower than expected thinning yield (fig.D), forced us to use the standard wafer as well. In the end, less than three-quarters of the installed chips turned out to be good. It is not clear whether the observed instability of some read-out channels (serving one quarter of the sensor matrix of each chip) is due to the chip or originates in the read-out system.

\subsection{Readout}

As each event contains $39 \mathrm{M}$ pixels, the raw data rate of the prototype is $61 \mathrm{~Gb} / \mathrm{s}$. Several FPGAs are used to manage this, as described in [W]. Incoming trigger bits are time-stamped with the read-out clock and stored separately. A 4 GByte local buffer memory allows to profit from the small duty factor of PS/SPS. It can store $0.55 \mathrm{~s}$ of pixel and trigger data. Transfer to the DAQ computer takes $\approx 100 \mathrm{~s}$ due to the software overhead of the TCP/IP protocol. Upon completion, the DAQ system waits for the next spill signal to store another $0.55 \mathrm{~s}$ of data. Note that the rolling shutter and chip read-out clocks are running continuously to keep a stable phase relation between all chips. From this continuous datastream the off-line processing reconstructs frames consisting of pixels read out maximum $640 \mu$ s before the trigger. A past/future protection of $640 \mu$ s prevents pile-up.

\section{Adjusting the discriminators}

Due to diffusion, the charge created by a particle will lead to a cluster of pixel hits. The size of the cluster will depend on the charge created by the particle (Landau distribution) and the threshold of the discriminators. For application in trackers the discriminators are usually adjusted such that an acceptable fake rate, measured as clusters not belonging to a track, is achieved. In the case of a calorimeter it is a priori not known whether the clusters will be well-separated, especially in the core of the shower. Therefore it was decided to use the number of hit pixels as a measure of the energy, instead of trying to derive the number of particles from the hit distributions. However this means that the discriminators should be set such that, in the absence of particles, an acceptable number of individual pixel hits is achieved, as opposed to clusters of hits. This is a more severe requirement that directly impacts on the signal-to-noise ratio of the energy signal. This is illustrated in fig.[1, which shows the measured response for $2 \mathrm{GeV}$ electrons. The noise level of 250 hits corresponds

\footnotetext{
${ }^{1}$ AMS-C35B4 OPTO by Austria Microsystems.
} 
to a noise hit fraction of $10^{-5}$. To achieve this, for some chips the threshold had to be set near the MPV peak, like chip 3 in fig.⿴囗十, so part of the signal is lost. One of the aims of the beam tests was to investigate how this influences the linearity and resolution. This work is still in progress.

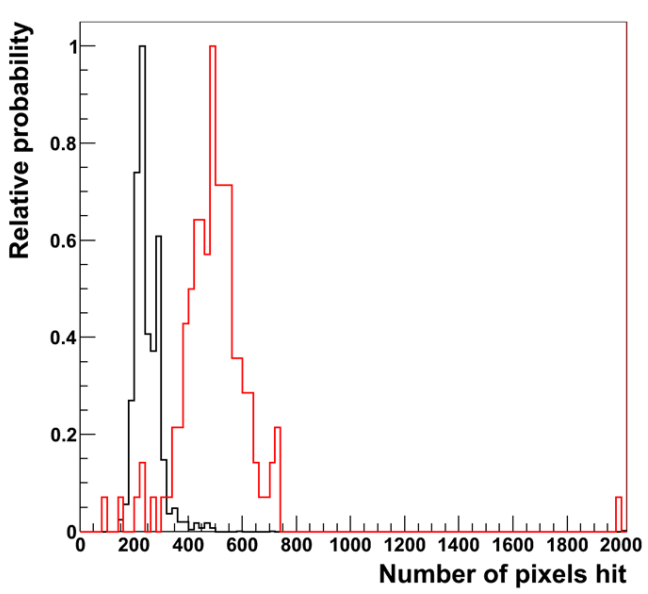

Figure 3: Distribution of pixel hits. Signal (red) compared to noise (black). Data taken at DESY.

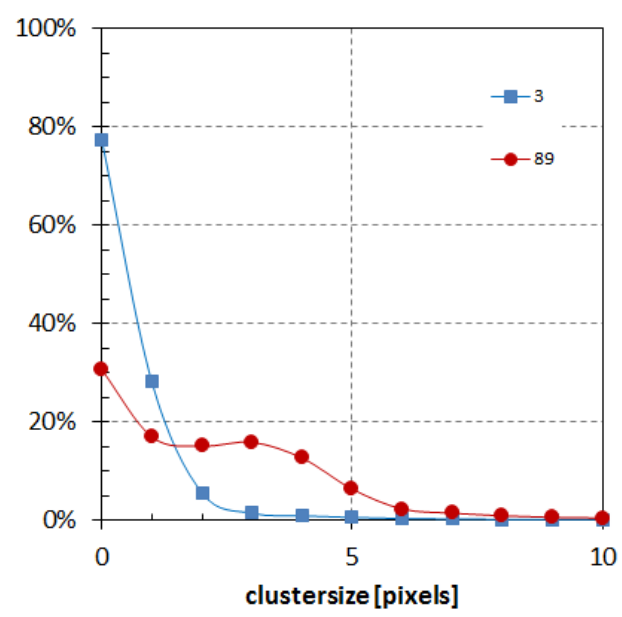

Figure 4: Clustersize histograms for two chips (numbers 3 and 89), collected from pion tracks.

\section{Performance}

The performance of the calorimeter was tested with beams of electrons and pions at DESY ${ }^{2}$ and CERN, at momenta of 2 up to $200 \mathrm{GeV} / c$. In an experiment, particle densities and the presence of other particle types will influence the performance, but this was not the topic of the present tests.

\subsection{Particle identification}

To demonstrate the particle identification capabilities we show the response to single $200 \mathrm{GeV} / \mathrm{c}$ particles: a positron, a non showering pion, behaving as a MIP particle, and a showering pion. Fig. 1 shows the hits of corresponding pixels of all layers projected onto the X-Y plane. The difference in shape between the two types of shower is clear and even the MIP signal stands out above the noise. From these uncalibrated, not aligned data one derives a Moliere radius of $11 \mathrm{~mm}$.

The three-dimensionall shower development is shown in figure 6 where the hits were integrated over rings centered on the presumed shower core for individual layers. The profiles tend to flatten out to $1 \mathrm{~cm}^{-2}$, corresponding to the noise level. The non showering pion (centre) shows a track-like profile whose apparent width is caused by the lack of precise alignment between the layers. One derives a misalignment of $\approx 0.1 \mathrm{~mm}$, several pixels. Using such tracks alignment to the sub-pixel level should be achievable. The profile of the positron (left) shows the typical shape expected for an electromagnetic shower. In the early layers the particle density in the centre is very high: the core of the shower. After the shower maximum the core gets depleted as no new particles are generated and the shower becomes wider. The hadronic shower (right) has no core and is wide from its start.

\footnotetext{
${ }^{2}$ The detector used at DESY had a different composition, with only 19 active layers and a total depth of $23 X_{0}$.
} 

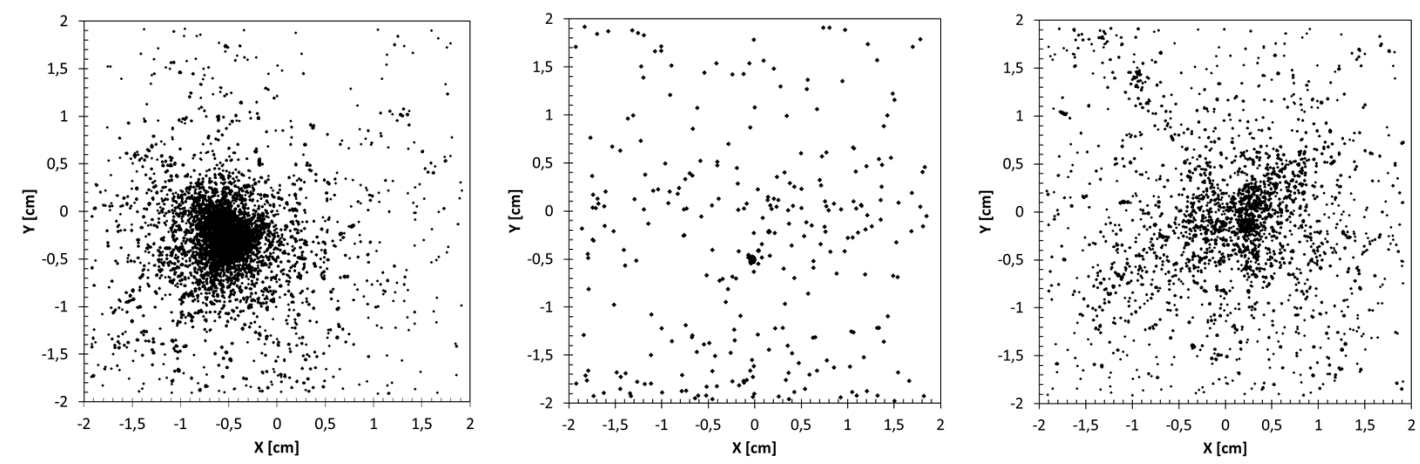

Figure 5: Projection of all hits generated by left: a $200 \mathrm{GeV} / c$ positron, centre: a $200 \mathrm{GeV} / \mathrm{c}$ non showering pion and right: a $200 \mathrm{GeV} / c$ showering pion.
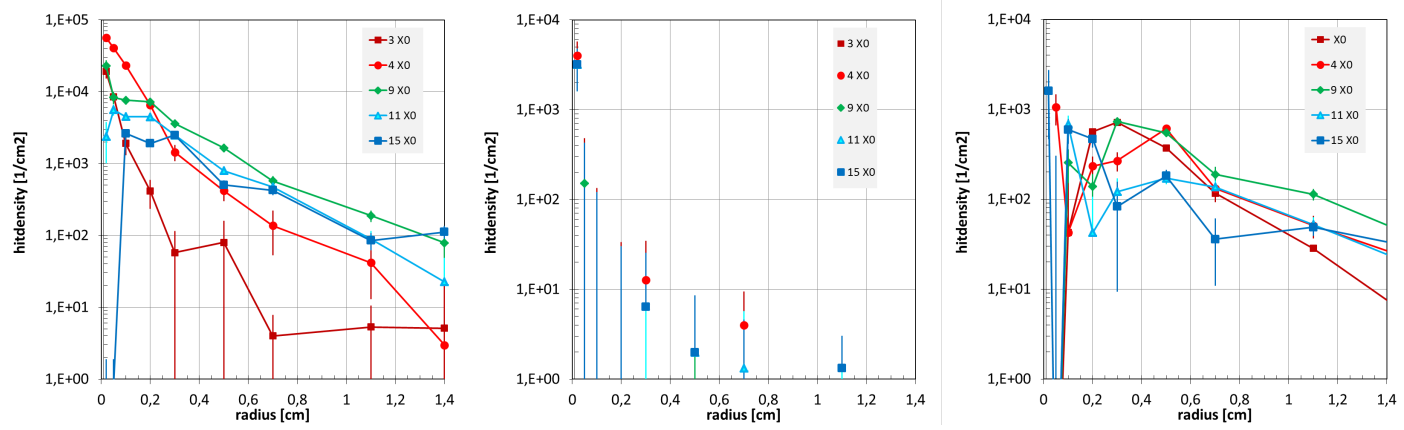

Figure 6: Lateral profiles at various depths of the hits generated by left: a $200 \mathrm{GeV} / c$ positron, centre: a $200 \mathrm{GeV} / c$ non showering pion and right: a $200 \mathrm{GeV} / c$ showering pion.

\subsection{Linearity and resolution}

The response of the uncalibrated detector (i.e. the number of pixels above threshold) is shown in fig.】, left. For comparison the number of particles according to $N=E / E_{c}$ (Rossi [ए2]), with $E_{c}=8.2 \mathrm{MeV}$, is also shown. As each particle produces a cluster of hits, one expects a fixed ratio between the two for an ideal, calibrated detector. The datapoints at 2 and $5 \mathrm{GeV}$ were scaled to account for the difference in the detectors during these measurements. This scaling appears to be not correct.

The resolution of the uncalibrated detector (work in progress) is shown in fig.[, right. Also shown are the results of a GEANT simulation of the detector: "all chips" is based on the perfect detector with all chips working, whereas "working chips" is based the actual detector state during the respective measurements. One can see the influence of "holes" in the calorimeter due to the $\approx 25 \%$ non-working chips.

\subsection{Special features}

In most of the datasets we see clusters of pixels arranged in either lines, circles or even discs of sizes up to $1 \mathrm{~mm}$. Figure 8 gives typical examples generated by $200 \mathrm{GeV} / \mathrm{c}$ positive particles, but they are also seen with $2 \mathrm{GeV}$ electrons. They seem to be related to showers and are never seen associated with single tracks. Their origin is as yet unknown. 

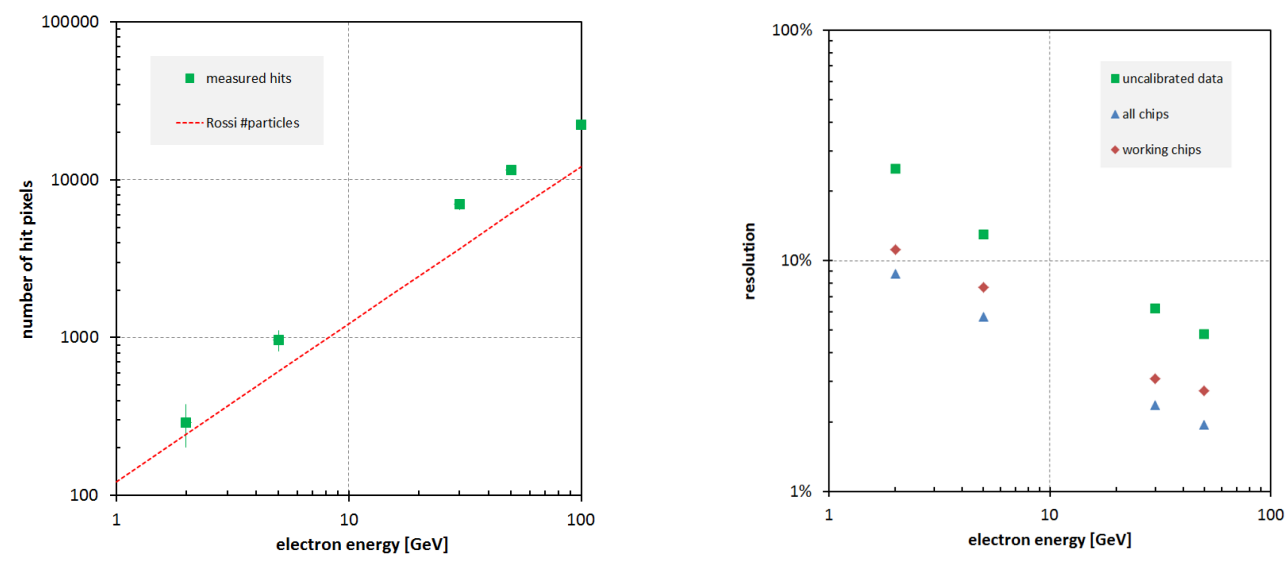

Figure 7: Left: The number of hits in the full detector, the line "Rossi" shows the theoretical number of particles. Right: Measured resolution of the uncalibrated detector (squares). The results of simulations of the ideal detector (triangles) and the real detector where only the signals from working chips were used (diamonds).
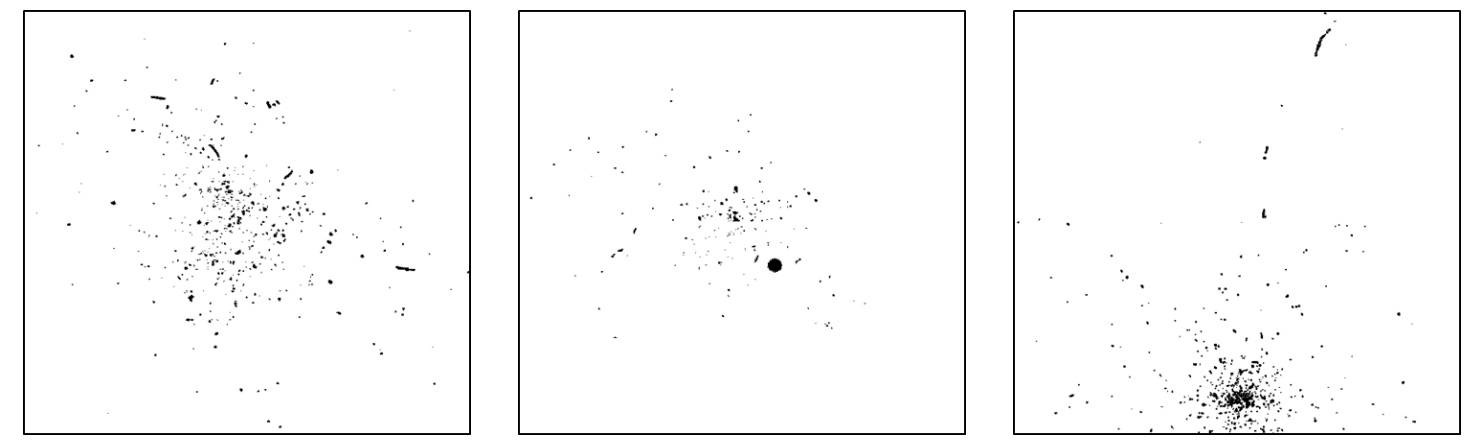

Figure 8: Patterns of hits arranged in lines (left), a disc (centre) and a curved line (right). The plots represent $2 \mathrm{~cm} * 2 \mathrm{~cm}$.

\section{Conclusion}

A prototype EM calorimeter with fine sampling and pixel counting has been built and successfully tested with particle beams. Its small Moliere radius of $11 \mathrm{~mm}$ combined with the very fine sampling will enable the direct separation of close showers without the need for unfolding. The data show the lateral profiles of showers in unprecedented detail, reveiling as yet unexplained features. The calibration still needs to be improved, this work is in progress.

\section{References}

[1] Boezio et al., A high granularity imaging calorimeter for cosmic-ray physics, Nucl. Inst. Meth. A487 (2002), 407.

[2] R. Turchetta et al., A monolithic active pixel sensor for charged particle tracking and imaging using standards VLSI CMOS technology, Nucl. Inst. Meth. A458 (2001), 677.

[3] P.F. Dauncey, Performance of CMOS sensors for a digital electromagnetic calorimeter, PoS (ICHEP 2010) 502 
[4] G. Voutsinas et al., Nuclear Physics B (Proc.Suppl.) 215 (2011) 48

[5] J.D. Segal et al., Second generation monolithic full-depletion radiation sensor with integrated CMOS circuitry, SLAC-PUB-14318 (2010)

[6] M. Szelezniak, CMOS pixel vertex detector for STAR, PoS (VERTEX 2008) 032

[7] ALICE ITS collaboration. Upgrade of the Inner Tracking System - Conceptual Design Report. CERN-LHCC-2012-013, 2012.

[8] G. Nooren, Extremely fine grained electro-magnetic calorimeter, PoS (RD11) 026

[9] Th. Peitzmann, Prototype studies for a forward EM calorimeter in ALICE, Proceedings of CHEF 2013

[10] A. Brogna et al., Manual PHASE1, IPHC Strasbourg

[11] D. Fehlker et al., Electronic for a highly segmented electromagnetic calorimeter prototype, 2013 JINST 8 P03015

[12] Rossi, High-energy Particles, Prentice-Hall,New York 1952 